\title{
Assessment Of Attitude Of Liver Transplantation Among Patients With Chronic Liver Disease
}

${ }^{*}$ Priya Thomas ${ }^{1}{ }^{* *}$ Akhil . C.S, Ameena Thaha ${ }^{* * *}$ Anjana . M.S

\begin{abstract}
Objectives- To identify the attitude among patients with chronic liver disease towards liver transplantation. Method- descriptive research design. Convenience sampling technique Result- $86.7 \%$ of chronic liver disease patients had average attitude towards liver transplantation and $13.3 \%$ of chronic liver disease patient had good attitude towards liver transplantation. Conclusion-The patients are lacking in-depth knowledge and awareness regarding the positive effects and quality of life changes after transplantation.
\end{abstract}

Key Words: Chronic liver disease, Attitude, Liver transplantation

\section{INTRODUCTION :}

Liver transplantation is the implantation of a liver from a donor into a recipient. Liver transplantation is indicated for the patient with chem.-hepatic cellular diseases ,metabolic liver disease, primary hepatic carcinoma and acute fulminant liver disease. ${ }^{1}$

The first human liver transplant was performed in 1963 by surgical team led by Dr.ThomasStarzl of Denver, Colorado, United states. The first liver transplant in Kerala was performed at Amrita Institute Of Medical Science in 2004. Attitudes are cognitive beliefs or emotions related to certain object or event or interventions. These attitude is influenced by various psychosocial background factors like personality traits or environmental characteristics and may affect together with an individual's subjective norms and perceived behavioral control. ${ }^{2}$ In transplantation medicine, the knowledge of attitude towards the transplantation experience influence the patient's intentions to follow the doctor's recommendations or patient adherence behavior

\section{Need for the study :}

The quality of life after a liver transplantation improves significantly and remains stable over a fairly long period of time. Attitude towards the liver transplantation have previously been investigated as primarily connection with organ donation or the carrying. The attitude of patient underscore the importance of health behaviour and medication adherence. Keeping in mind about the importance of liver transplantation, the investigators was motivated to conduct the study to evaluate the attitude of patient with chronic liver disease towards liver transplantation. ${ }^{3}$

\section{Statement of the problem}

"A study to assess the attitude towards liver transplantation among patients with chronic liver disease admitted at AIMS Kochi." 


\section{OBJECTIVES :}

- Assess the attitude among patients with chronic liver disease towards liver transplantation.

- Find the association between attitude and selected demographic variables

\section{RESEARCH METHODOLOGY:}

Non-experimental descriptive research design with quantitative research approach

Was adopted for the study. The study was conducted in medical and surgical wards of AIMS KOCHI. The samples was collected by using Non-convenience sampling method. It consists of patient with chronic liver disease 30 patients suffering from liver disease patients were taken as per the sample selection criteria. Sample size was determined by using the formula $4 \mathrm{pq} / \mathrm{d} 2$.

Data collection Instruments and Technique

Data was collected by using two tools developed by the investigator.

Tool: A self-administered questionnaire to collect demographic variables and selfadministered attitude scale towards liver transplantation among patient with chronic liver disease

Section A: Socio-demographic data.

It includes seven items regarding age, sex, marital status, educational qualification, income, occupation, religion

Section B: Clinical data and self -administered attitude scale Clinical data includes 4items regarding period of illness, history of alcoholism, history of smoking, weight . Selfadministered attitude scale includes 20 statements regarding liver transplantation.

These statements were scored as strongly disagree, disagree, uncertain, agree, strongly agree.

RESULT:
The first objective was to assess the attitude among patients with chronic liver disease towards liver transplantation.

The recent study shows that majority of chronic liver disease patients had negative attitude 26(87\%) towards liver transplantation. and only few had $4(13 \%)$ positive attitude.

The second objectives of the study were to find out the association between the level of stress and selected demographic variables.

The association was done using Man Whitney test. The study reveals that patient with history of alcoholism had good attitude towards liver transplantation but patient with no history of alcoholism has average attitude towards liver transplantation. The study findings were supported by the study conducted by Sinead Waldron regarding attitude towards liver transplant patient with alcoholic liver disease; the influence of prosocial personality and patient information. One hundred and eighty eight participants (105 females and 83 males) completed batteries of scales that measured organ donation willingness and pro-social personality levels. Participants rated the patient with history of alcohol abuse who was no longer drinking as a significantly lower priority for transplant surgery than a patient with a genetic liver condition. This implied that patients with alcoholic liver disease perceived as less deserving of liver transplants.

\section{CONCLUSION :}

Chronic liver disease is one of the major health problem in our country and also most of the cases undergo the liver transplantation. 


\section{Pondicherry Journal of Nursing}

So assessment of attitude towards liver transplantation among chronic liver disease patients are very essential. Majority of the patient have only average attitude but few have good attitude towards liver transplantation.

\section{REFERENCES :}

1. Strazl TE, Groth CG, Brettschneider L, etal.Orthotopic homotransplantations of the human liver.1968;168:392-415
Vol 9 Issue 3 SEP-DEC 16

transplanted liver patients : November 2003:3-33

3. K. Strong .C.Mathers ,S.Leeder. Preventing chronic diseases: how many lives can we save lancet,366(2005),pp.1578-1582

4.Shimaa Arafat Abdel- Monem: Attitude towards liver donation and transplantation.nhttp://eluc.edu.eg/eulcv5/li braries/thesis $=11894810$.

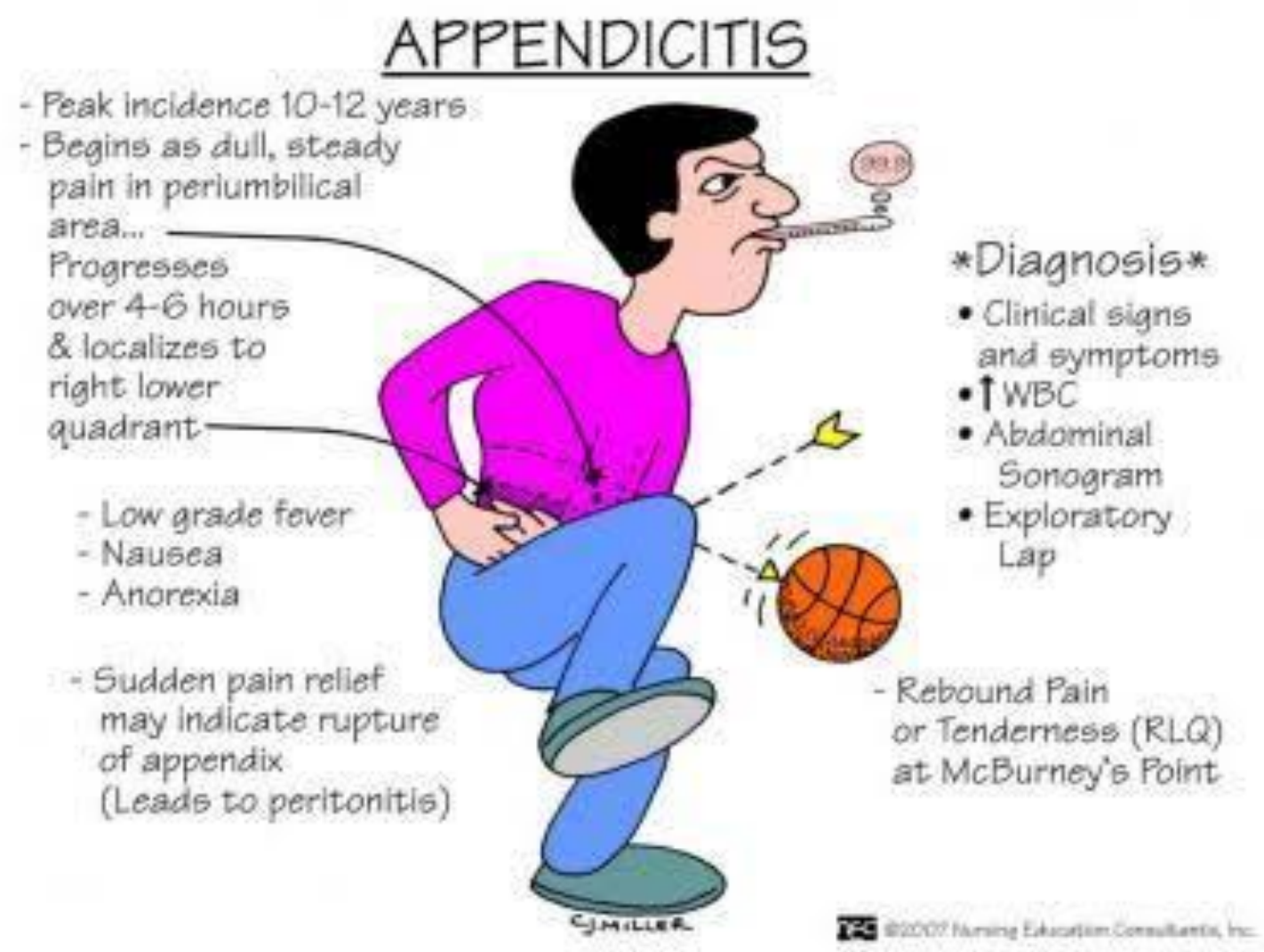

2. Van der PlasS.Hansen B. de Boer J.et al, Generic and specific health related quality of life in non-cirrhotic, cirrhotic and 